\title{
Reverse Polarized Inductive Coupling to Transmit and Receive Radiofrequency Coil Arrays
}

\author{
Haydar Celik ${ }^{1,2}$ and Ergin Atalar, ${ }^{1,2 *}$
}

In this study, the reverse polarization method is implemented using transmit and receive arrays to improve the visibility of the interventional devices. Linearly polarized signal sourcesinductively and receptively coupled radiofrequency coils-are used in the experimental setups to demonstrate the ability of the method to separate these sources from a forward polarized anatomy signal. Two different applications of the reverse polarization method are presented here: (a) catheter tracking and (b) fiducial marker visualization, in both of which transmit and receive arrays are used. The performance of the reverse polarization method was further tested with phantom and volunteer studies, and the results proved the feasibility of this method with transmit and receive arrays. Magn Reson Med 67:446-456, 2012. (C) 2011 Wiley Periodicals, Inc.

Key words: reverse polarization; transmit array system; phased array coils; interventional MRI; inductive and receptive coupling; interventional device tracking; fiducial marker

In a previous study, the reverse polarization method for catheter tracking using a birdcage coil was introduced (1), and the method has been used for different applications by other groups $(2,3)$. This method separates anatomical information from an inductively coupled radiofrequency (ICRF) coil coupled to an external coil, during signal reception only. Note that an ICRF decoupled from the transmit field using back-to-back diodes is called a receptively coupled $R F(R C R F)$ coil $(1,4,5)$. Although this method can be an excellent candidate for making interventional devices, such as catheters, guidewires, biopsy needles, visible, its main drawback is the requirement of a birdcage volume coil as the receiver. In today's practice, birdcage coils are seldom used as receive coils because of their lower performance compared with the phased array coils (6). Notably, it is known that individual simple elements of a phased array coil create a linearly polarized magnetic field (7); therefore, it is not a trivial task to generate reverse polarized sensitivity using the phased array coils.

Here, a method is presented for obtaining reverse polarized sensitivity using a phased array coil system. It is also shown that by using duality, the proposed method can be employed to obtain reverse polarization through RF transmit-array systems.

${ }^{1}$ National Research Center for Magnetic Resonance (UMRAM), Bilkent University, Ankara, Turkey.

${ }^{2}$ Department of Electrical and Electronics Engineering, Bilkent University, Ankara, Turkey.

${ }^{*}$ Correspondence to: Ergin Atalar, Ph.D., UMRAM, Cyberplaza, Block C, 2nd Floor, Cyberpark, Bilkent University, Bilkent, Ankara, Turkey TR-06800. E-mail: ergin@ee.bilkent.edu.tr

Received 4 October 2010; revised 25 April 2011; accepted 5 May 2011. DOI 10.1002/mrm.23030

Published online 7 June 2011 in Wiley Online Library (wileyonlinelibrary. com).

(c) 2011 Wiley Periodicals, Inc.
"Wireless" active catheter tracking (4) and fiducial marker visualization (8-12) are demonstrated as applications of the proposed method. In both of these applications, induced RF current flows on a coupled RF (CRF) coil, amplifies the rotating magnetization vector, and finally turns into an oscillating magnetization in a linear path. This magnetization can be decomposed into rotating magnetization vectors, one traveling in the forward direction and the other in reverse. As an anatomical signal can only be generated by the forward polarized magnetization vector, an image that is sensitive to the reverse polarized magnetization will solely contain the signal received by the CRF coil.

\section{THEORY}

The reverse polarization method can be implemented using the receive and/or transmit paths.

\section{Reverse Polarization Method Using Receive (Phased)} Array RF Coils

Implementing the reverse polarization method to a receive coil with uniform sensitivity, such as the birdcage coil, is trivial because: (i) both of the feeds of a birdcage coil have the same sensitivity magnitude and (ii) the phase difference between the feeds is the same for all pixels. As a result, shifting the phase of the $y$-channel by $-90^{\circ}$ or $90^{\circ}$ and summing with the $x$-channel produce forward or reverse polarized modes of images $(1,13)$. However, phased array coils do not have uniform sensitivity profiles and thus to obtain a reverse polarized mode of image, a more sophisticated algorithm is necessary. In this section, anatomy and CRF coil signals received by a linear phased array coil will be derived. Additionally, the algorithm of the reverse polarization method for receive array coils, which consist of linear coils will be presented.

If the direction of the main magnetic field of an MR scanner is reversed, a quadrature receive-only birdcage coil cannot receive any signal. On the other hand, if a linear coil, such as a simple loop coil, is used to receive the signal, the direction of the magnetic field is not important. In either case, the linear coil picks up the signal with equal sensitivity. In theory, it is possible to obtain pure forward or reverse polarization at a point of interest by adjusting the phase and amplitudes of signals received by the linear coil elements, but, sensitivity information of coil elements has to be known perfectly. Any error in the sensitivity information may lead to error in the field polarization. In reality, the error can be reduced by increasing number of elements. 
In the MR literature, the rotation direction of a magnetic field is usually explained in the rotating frame. Because both forward and reverse polarizations will be utilized in this study, it is difficult to explain this concept in the rotating frame. Therefore, in this text, magnetic fields will be expressed using the phasor notation with the $e^{i \omega_{0} t}$ convention, where " $i$ " is the imaginary unit number $(\sqrt{-1})$ and $\omega_{0}$ is the Larmor frequency.

Assume that a conventional body coil is used for excitation and a phased array coil with $N$ linear coil elements is used to image an anatomy with a CRF coil placed inside it. There are two different coupling mechanisms when a CRF coil is used in MRI (1). The first one is transmit coupling, which occurs between the transmit coil and the CRF coil. The second type of coupling occurs during reception. The ICRF coil is exposed to both transmit and receive couplings, on the other hand, the RCRF coil is decoupled from the transmit field. In this section of the theory, signals are derived due to receive phase. Therefore, the phased array coil elements receive two types of signals from a point of interest close to the CRF coil: a direct signal of the spins and an indirect signal of the CRF coil. Rotating spins induce a current on the CRF coil and this current creates a linear magnetic field that has both forward and reverse polarized components. The signal of the spins and then the signal of the CRF coil will be derived.

\section{Anatomy Signal}

Let $v_{n}$, anatomy be the open circuit voltage of the $n$th element of an $N$-channel phased array coil caused by the spins at the point of interest. To find the total voltage, this quantity needs to be integrated over the whole volume. However, by itself, this value is directly related to the sensitivity of the coil element at this point of interest. The voltage can be formulated using the reciprocity principle (14-16):

$$
V_{n, \text { anatomy }}=-i \omega_{0} \vec{M} \cdot \vec{B}_{n},
$$

where "." represents the dot product and $\vec{M}$ is the phasor representation of the rotating magnetization vector given by:

$$
\vec{M}=m\left(\widehat{a}_{x}+i \widehat{a}_{y}\right),
$$

where $m$ is the complex representation of the transverse magnetization and, $\widehat{a}$, and $\widehat{a}_{y}$ are unit vectors in $x$ and $y$ directions, respectively. Note that Eq. 2 is the phasor equivalent of the time domain representation of the magnetization $\vec{M}(t)=|m| \cos \left(\omega_{0} t+\angle m\right) \hat{a}_{x}-|m| \sin \left(\omega_{0} t+\angle m\right) \widehat{a}_{y}$. In Eq. $1, \vec{B}_{n}$ is the magnetic field at the point of interest generated by the $n$th element of the receive phased array coil when a unit current is applied to the terminals of the coil element (Fig. 1a). Note that $\vec{B}_{n}$ is assumed to be a linearly polarized magnetic field for the sake of simplicity and understandability without sacrificing generality. Also, all the elements of the phased array coil are assumed to have the same phase at a point of interest because this value is carefully calibrated in the MRI scanner. In this study, the z-component of the magnetic field is ignored as it has no contribution to this analysis.

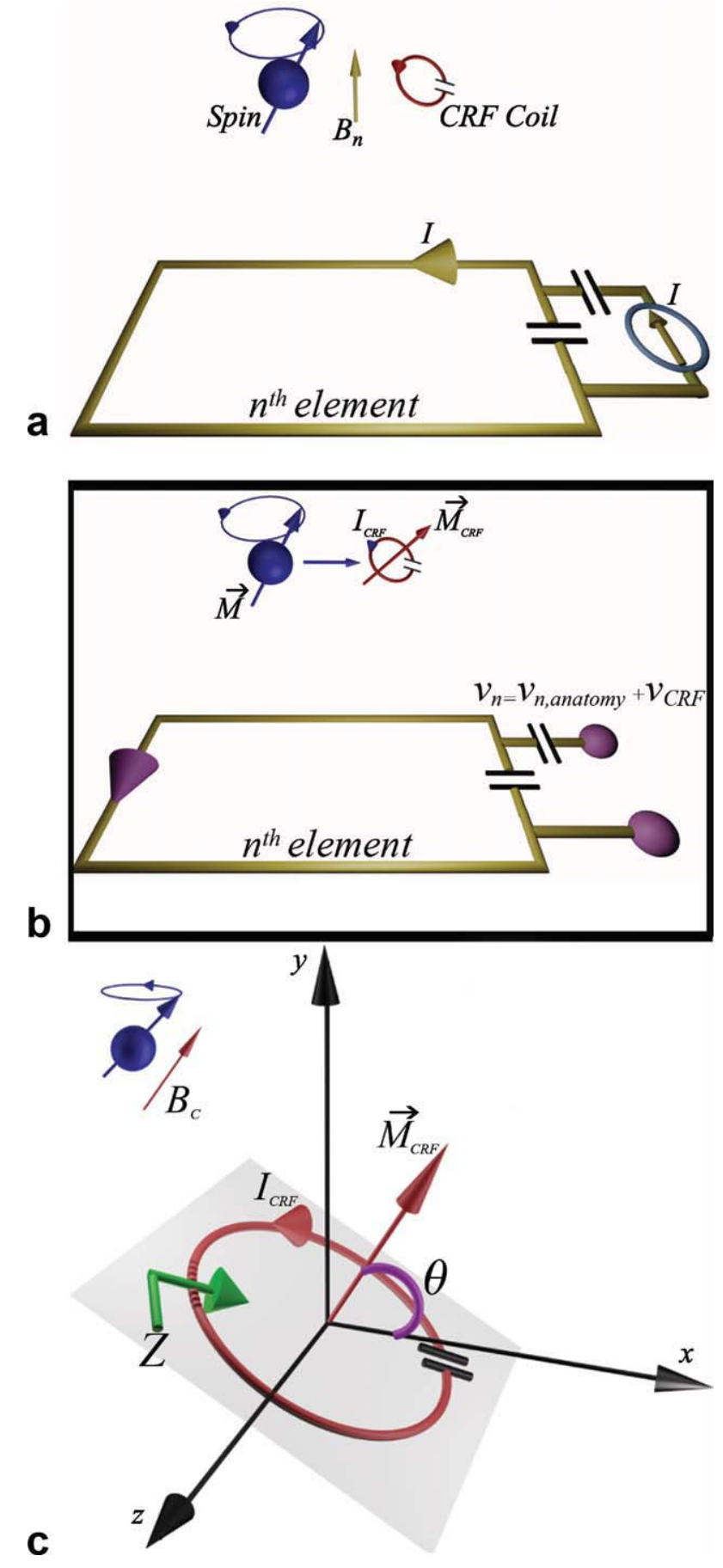

FIG. 1. Sketch of the $n$th element of the phased array coil, CRF coil, and spin interactions. a: $\vec{B}_{n}$ is the magnetic field generated by the $n$th element of the receive phased array coil at the point of interest when the unit current, $l$, is applied to the terminals of the coil element. b: $\vec{M}$ is the rotating magnetization vector of spins and $\vec{M}_{\mathrm{CRF}}$ is the magnetization vector of the CRF coil that is oscillating in a linear trajectory. The spins around the CRF coil induce a current, ICRF. This current on the small CRF coil can be represented by a magnetization vector, $\vec{M}_{\mathrm{CRF}}$. As a result of these magnetizations, a voltage, $v_{n}$, is induced on the terminals of the external coil. c: $\vec{B}_{C}$ is the magnetic field generated by the CRF coil at a point of interest when a unit current, $l$, is applied to an imaginary terminal of the coil. $\theta$ is the orientation angle of with respect to $x$-axis of the CRF coil, and $Z$ is the impedance at this imaginary terminal of the CRF coil. 


\section{CRF Coil Signal}

A CRF coil is the secondary source of the magnetic field, i.e., the spins around the CRF coil induce the current per unit volume, $I_{\mathrm{CRF}}$, on the CRF coil. This current on the small CRF coil can be represented by a magnetization oscillating on a linear trajectory (Fig. 1b). Note that a linearly polarized magnetization can be decomposed into two counter-rotating magnetizations, i.e., forward and reverse polarized magnetizations. Linearly polarized external receive coils are sensitive to both components of the linearly polarized magnetization.

The current per unit volume on a small CRF coil can be modeled using a magnetization vector in the direction of the surface normal vector as (Fig. 1c):

$$
\begin{aligned}
\vec{M}_{\mathrm{CRF}} & =I_{\mathrm{CRF}} s\left(\cos \theta \widehat{a}_{x}+\sin \theta \widehat{a}_{y}\right) \\
& =I_{\mathrm{CRF}} s\left[e^{i \theta}\left(\widehat{a}_{x}-i \widehat{a}_{y}\right)+e^{-i \theta}\left(\widehat{a}_{x}+i \widehat{a}_{y}\right)\right] / 2
\end{aligned}
$$

where $\theta$ is the orientation angle of the CRF coil with respect to the $x$-axis.

Here, an important property of the CRF coil is that its magnetization vector can be decomposed into forward and reverse polarized components of equal magnitude:

$$
\vec{M}_{\mathrm{CRF}}=\vec{M}_{\mathrm{CRF}, \mathrm{f}}+\vec{M}_{\mathrm{CRF}, \mathrm{r}},
$$

where

$$
\begin{aligned}
& \vec{M}_{\mathrm{CRF}, \mathrm{f}}=s I_{\mathrm{CRF}} e^{-i \theta}\left(\widehat{a}_{x}+i \widehat{a}_{y}\right) / 2 \\
& \vec{M}_{\mathrm{CRF}, \mathrm{r}}=s I_{\mathrm{CRF}} e^{i \theta}\left(\widehat{a}_{x}-i \hat{a}_{y}\right) / 2
\end{aligned}
$$

Similar to the anatomy case in Eq. 1, the voltage induced on the $n$th coil by a CRF coil can be expressed using the magnetization vector of the CRF coil, as (Fig. 1b):

$$
V_{n, \mathrm{CRF}}=-i \omega_{0} \vec{M}_{\mathrm{CRF}} \cdot \vec{B}_{n} .
$$

Note that the magnetic field, $\vec{B}_{n}$, is assumed to be the same magnetic field as in Eq. 1, because the CRF coil is small and the point of interest is very close to it.

Integrating Eq. 4 into Eq. 6 results in:

$$
V_{n, \mathrm{CRF}}=-i \omega_{0}\left\{\left[\vec{M}_{\mathrm{CRF}, \mathrm{f}}+\vec{M}_{\mathrm{CRF}, \mathrm{r}}\right] \cdot \vec{B}_{n}\right\} .
$$

The induced current on the CRF coil can be calculated using a method similar to the derivation of the anatomy signal. The main difference is that the CRF coil has no terminal; therefore; the induced current is calculated instead of the voltage. Assume that $\vec{B}_{C}$ is the magnetic field generated by the CRF coil at a point of interest when a unit current is applied to an imaginary terminal of the coil (Fig. 1b); then the induced current on the CRF coil due to the magnetic resonance phenomenon is:

$$
I_{\mathrm{CRF}}=-i \omega_{0} m B_{C} e^{i \theta} / Z
$$

where $Z$ is the impedance at this imaginary terminal (Fig. 1c). As a result, $V_{n, \mathrm{CRF}}$ can be expressed in terms of $V_{n \text {,anatomy: }}$ :

$$
V_{n, \mathrm{CRF}}=m S_{n} C_{\mathrm{f}}+m S_{n}^{*} C_{\mathrm{r}},
$$

where $S_{n}$ is the sensitivity of the $n$th element and defined as $S_{n}=V_{n \text {,anatomy }} / m$. Here, “*” is the conjugation operator and $C_{\mathrm{f}}$ and $C_{\mathrm{r}}$ are the forward and reverse polarization amplification factors, respectively:

$$
\begin{aligned}
& C_{\mathrm{f}}=-i \omega_{0} s B_{c} / 2 Z \\
& C_{\mathrm{r}}=i \omega_{0} s e^{i 2 \theta} e^{i 2 \phi} B_{c} / 2 Z
\end{aligned}
$$

$\phi$ is the phase of $\vec{B}_{n}$. Note that there is only a phase difference between these values.

Finally, the total voltage can be expressed by the sum of the anatomy and CRF coil voltages:

$$
\begin{aligned}
v_{n} & =v_{n, \text { anatomy }}+v_{n, \mathrm{CRF}} \\
& =S_{n}\left(m c_{\mathrm{f}}+m\right)+S_{n}^{*} m c_{\mathrm{r}}
\end{aligned}
$$

As a result, the matrix form of the above equation is:

$$
\left(\begin{array}{c}
v_{1} \\
v_{2} \\
\vdots \\
v_{N}
\end{array}\right)=\left(\begin{array}{cc}
S_{1} & S_{1}^{*} \\
S_{2} & S_{2}^{*} \\
\vdots & \vdots \\
S_{N} & S_{N}^{*}
\end{array}\right) \cdot\left(\begin{array}{c}
m+m c_{\mathrm{f}} \\
m c_{\mathrm{r}}
\end{array}\right)
$$

\section{The Reverse Polarization Algorithm}

This manuscript deals with the challenge of extracting the reverse polarization component of a signal $m c_{\mathrm{r}}$ from the acquired data. By doing this, the position of a coupled coil placed on a catheter or on a body as a fiducial marker can be identified.

The vector on the left-hand side of the above equation is already known and represents pixel values on the images that are reconstructed using the signal received by each of the coil elements. To find $m c_{\mathrm{r}}$, the sensitivity values for each coil element must be known. Once the sensitivities of all coil elements are known, the reverse polarized component of the signal (together with the forward component as a byproduct) can be obtained by a matrix pseudo-inversion operation:

$$
\left(\begin{array}{c}
m+m c_{\mathrm{f}} \\
m c_{\mathrm{r}}
\end{array}\right)=\left(\begin{array}{cc}
S_{1} & S_{1}^{*} \\
S_{2} & S_{2}^{*} \\
\vdots & \vdots \\
S_{N} & S_{N}^{*}
\end{array}\right)^{\dagger} \cdot\left(\begin{array}{c}
v_{1} \\
v_{2} \\
\vdots \\
v_{N}
\end{array}\right)
$$

where “†” represents a pseudo-inversion operator.

\section{Reverse Polarization Method Using Transmit Array Coils}

The algorithm of the reverse polarization method for the receive array coils is explained above. To tackle this problem, the reverse polarized signal needs to be calculated for each pixel of the image using a postprocessing algorithm. Using the reciprocity principle, one can conclude that the reverse polarization algorithm for a transmit array is a dual problem. However, unlike the receive arrays, a transmit operation cannot be conducted using postprocessing. Therefore, by using the above algorithm, the reverse polarization can only be achieved on a single point in space. To solve this issue, the algorithm can be generalized for multiple points, but the formulation indicates that reverse polarization can be obtained only on limited number of points 


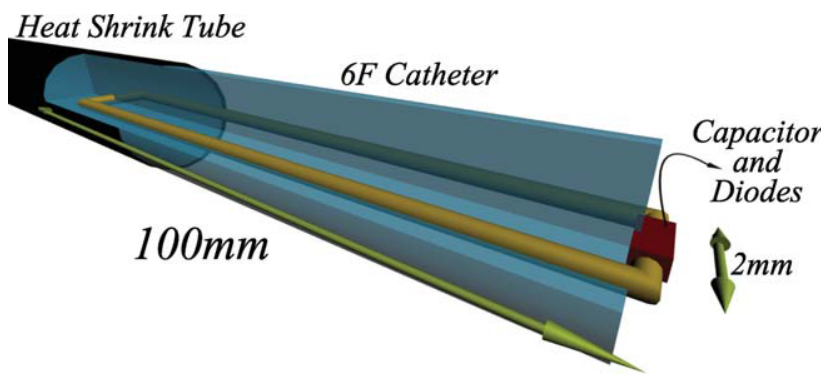

FIG. 2. The CRF coil design. The ICRF coil has a tuning capacitor, the RCRF coil has a pair of back-to-back diodes in addition to the tuning capacitor. [Color figure can be viewed in the online issue, which is available at wileyonlinelibrary.com.]

with an $N$-channel transmit array system. By properly distributing these points in a region of interest, one may obtain reverse polarization in that region.

Alternatively, a birdcage coil may be fed from its two quadrature ports independently as a transmit array coil. In this configuration, obtaining reverse polarized transmission is straightforward, because a postprocessing operation is not necessary $(1,2)$. Shifting the phase of the $y$-channel by $-90^{\circ}$ or $90^{\circ}$ with respect to the $x$-channel produces forward or reverse polarized excitation modes. A RF magnetic field does not excite spins if it is rotating in the "reverse" direction, but it induces a current on the CRF coil. Then, the secondary field created by the CRF coil excites spins in its vicinity. Therefore, when reverse polarized excitation is used, the anatomy signal will be suppressed and consequently the reconstructed image will consist of the CRF signal only. Note that with the help of the transmit array system, both forward and reverse (or linear) excitations are possible, and the forward or reverse polarization mode of an image can be obtained without postprocessing.

\section{METHOD}

In this study, a 3-T Siemens TIMTrio with an experimental eight-channel transmit array system was used. Siemens body matrix, spine matrix, and head matrix coils were used for signal reception. These coils use mode matrix combiners (17). The mode matrix combiners introduce phase to the coil elements and change weighting of them, so, coil linearities are disturbed. Also, Siemens coils have three different combination modes: CP (Circularly Polarized), Dual, and Triple. To reconstruct the linear polarized data, images were acquired in triple mode (17) and proper complex constants were multiplied with the element data and recombined. A MATLAB (Version 7.6; Mathworks Inc., Natick, MA) code was written for this purpose.

The receive coil sensitivity information is crucially important for the reverse polarization method. The sensitivity maps can be acquired using different methods (1821). In this study, these maps were obtained using the central part of the $k$-space matrix or spatial low-pass filtering. The filtering removes the effect of the CRF coil on the image because the coil is small, and its information is in the high-frequency components of the image However, one cannot obtain CRF coil data by high-pass filter- ing because anatomy also has high-frequency components. The sensitivity map window, which determines the area at the center of the $k$-space matrix, was adjusted according to the applications.

After obtaining the sensitivity maps, the reverse polarization factor for each pixel was calculated using the pseudoinverse command "pinv" of MATLAB. The reverse polarization mode of the image was color-coded and combined with the squared-summed image of the subject.

Only Siemens body coil was used for transmission in all experiments. For the first part of the study, phased array case, the conventional body coil excitation was applied. On the other hand, the quadrature hybrid was eliminated for the transmit array case, and two body coil channels were fed by the transmit array system. The transmit array system on site has eight RF channels and two of them used for transmit array experiments. Relative phases of the two channels had to be calibrated before the experiments as they change after each system reboot. By using the reverse polarization mode, two transmit channels were calibrated, i.e., the phase was adjusted to obtain the noisy reverse polarization mode of the image. In the ideal case, adding $90^{\circ}$ to the $y$-channel and running both channels create the reverse polarized field and no spins are excited. Because of the shift caused by the system reboot, the $y$-channel phase was scanned between $40^{\circ}$ and $140^{\circ}$ with an increment of $10^{\circ}$. The image with a minimum signal level was assumed to be the reverse polarized mode of the image. After fine tuning, the relative phase between the $x$ - and $y$ channels was found.

The proposed method was implemented for both catheter tracking and fiducial marker visualization applications.

\section{Catheter Tracking}

The ICRF coil was $100 \mathrm{~mm}$ long and constructed on a 6 $\mathrm{F}$ Teflon catheter using a coated copper wire $0.4 \mathrm{~mm}$ in diameter. A heat shrink tube was used for isolation, resulting in a prototype device with an outer diameter of $3 \mathrm{~mm}$ (9 F) (Fig. 2). It was tuned by a $22 \mathrm{pF}$ ceramic chip capacitor (ATC, Huntington Station, NY) to 123.23 $\mathrm{MHz}$ using an HP 8753D network analyzer (Agilent

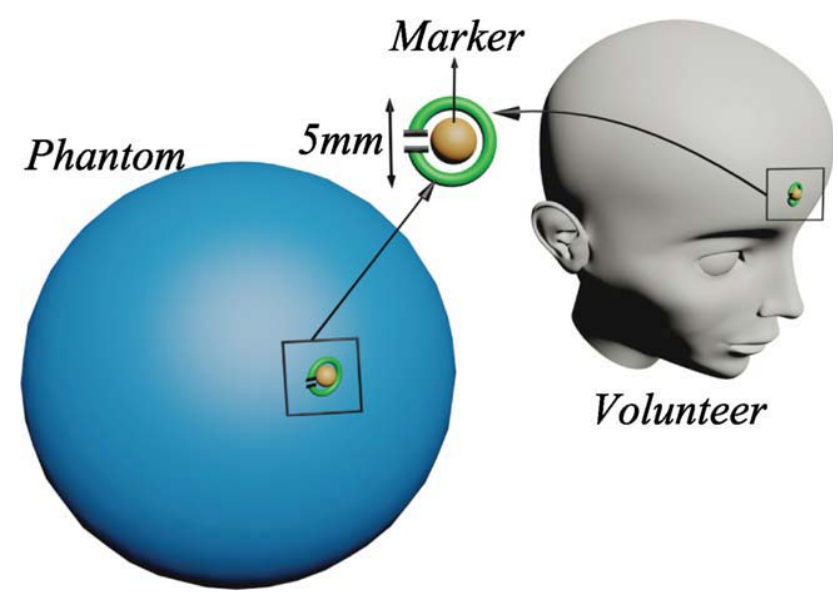

FIG. 3. Sketch of the $\mathrm{NaCl}$ solution phantom and a volunteer for fiducial marker visualization. [Color figure can be viewed in the online issue, which is available at wileyonlinelibrary.com.] 


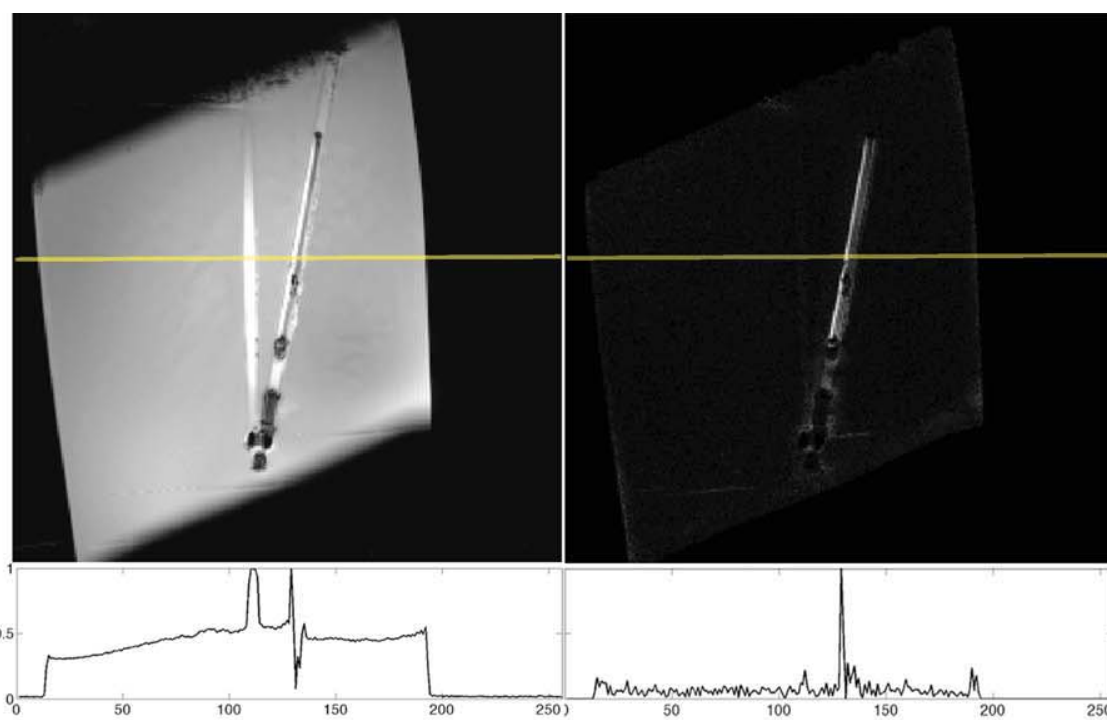

a

b

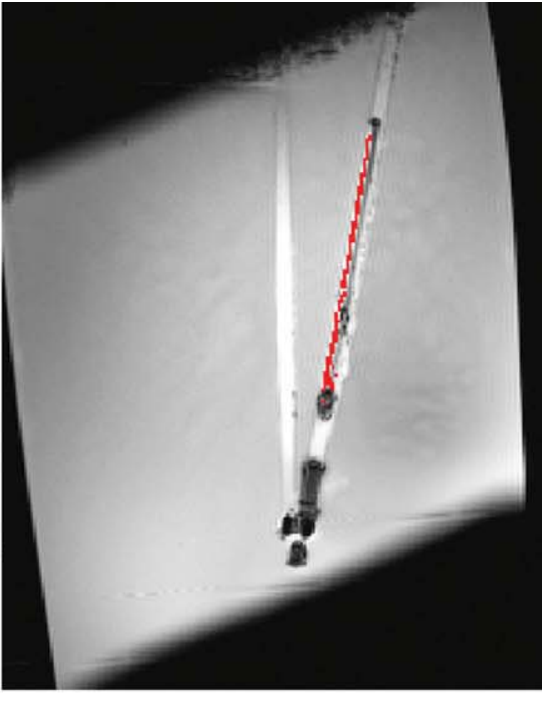

C

FIG. 4. Oblique images of the oil phantom, $\mathrm{KCl}$ solution filled straw (left), the RCRF coil (right), and the normalized performance plots (yellow line on the image) using the reverse polarization method for phased array coils. a: The forward polarization mode of the image and the performance plot at the bottom, (b) the reverse polarization mode of the image and the performance plot at the bottom, and (c) the colorcoded image. Although there is a $\mathrm{KCl}$ solution-filled straw with higher signal intensity than the RCRF coil as a disturber, the reverse polarization method can separate the RCRF coil from the phantom. The performance plots show the effectiveness of the method clearly. [Color figure can be viewed in the online issue, which is available at wileyonlinelibrary.com.]

Technologies, Santa Clara, CA). A pair of back-to-back MMBD7000LT1 switching diodes (Infineon Tech., Germany) in parallel to the $22 \mathrm{pF}$ tuning capacitors was used for the decoupling of the RCRF coils.

In the catheter tracking experiments, oil phantoms were used and images were acquired using the spine and body matrix phased array coils. Fast gradient echo sequence with the following imaging protocol is used: TR/TE: $40 / 3.4 \mathrm{~ms}$, flip angle: $40^{\circ}$, slice thickness: $5 \mathrm{~mm}$.

\section{Fiducial Marker Visualization}

The ICRF coil was constructed with three turns of copper wire of $0.5 \mathrm{~mm}$ in diameter, forming a circle with a diameter of $5 \mathrm{~mm}$ (Fig. 3). It was tuned by a $16 \mathrm{pF}$ ceramic chip capacitor (ATC, Huntington Station, NY) to $123.23 \mathrm{MHz}$ using an HP 8753D network analyzer (Agilent Technologies, Santa Clara, CA). In both the transmit and receive array phantom experiments, images were acquired using the head matrix phased array coil and fast gradient echo with the

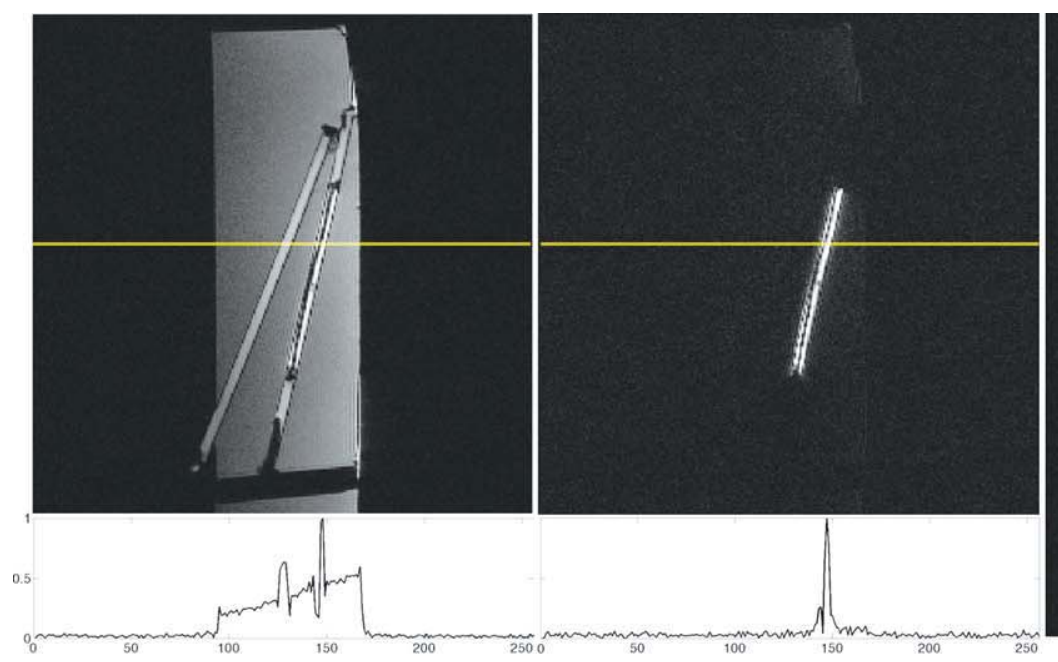

b

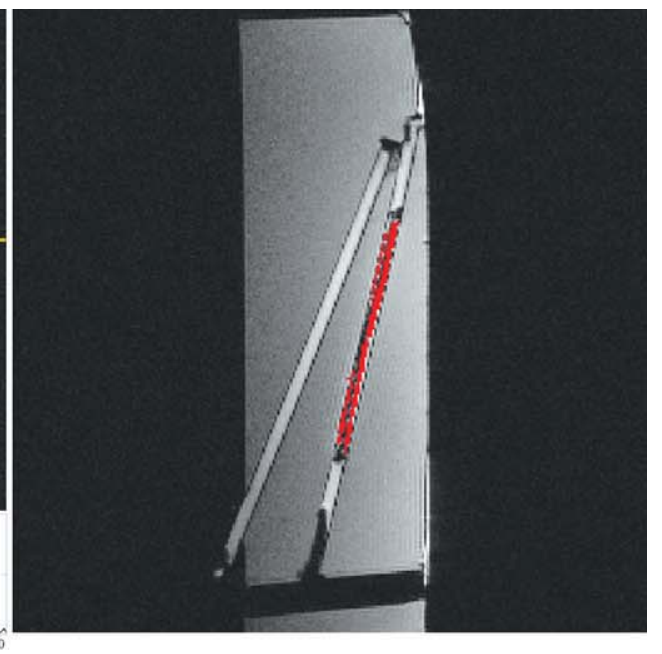

C

FIG. 5. Sagittal images of the oil phantom, KCl solution filled straw (left), the ICRF coil (right), and the normalized performance plots (yellow line on the image) using the reverse polarization method for transmit array system. a: The forward polarization mode of the image and the performance plot at the bottom, (b) the reverse polarization mode of the image and the performance plot at the bottom, and (c) the colorcoded image. Similar to the receive array case, suppression of the phantom and $\mathrm{KCl}$ filled straw signal for MR guidance is effective. [Color figure can be viewed in the online issue, which is available at wileyonlinelibrary.com.] 


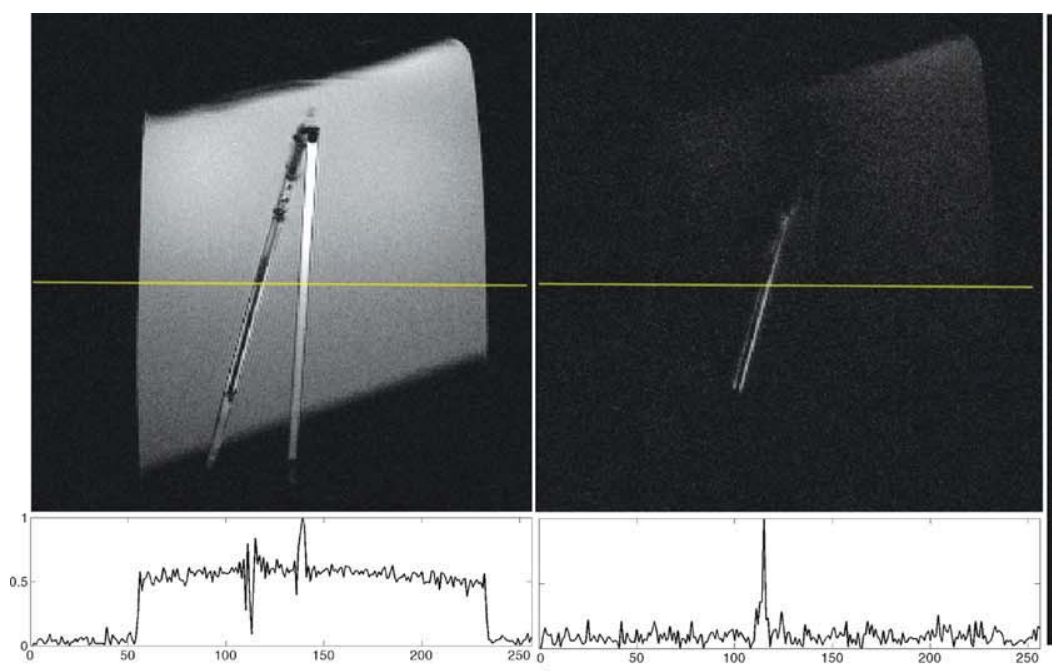

a

b

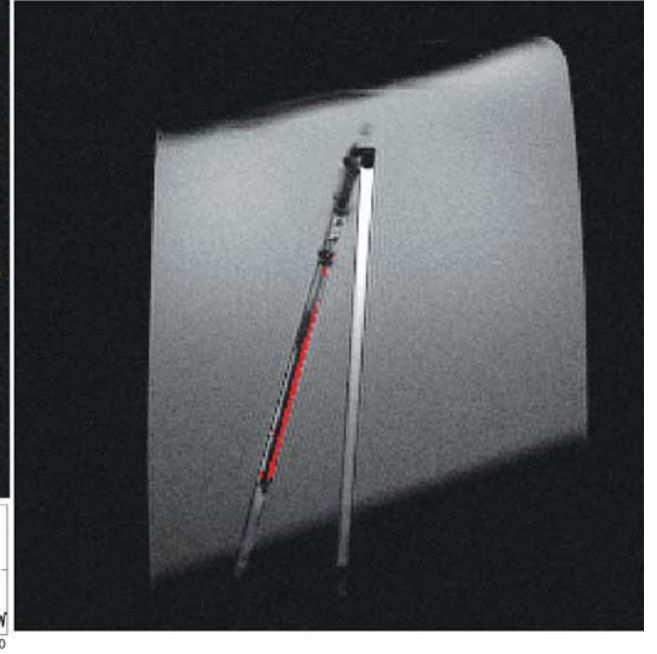

C

FIG. 6. Oblique images of the oil phantom, $\mathrm{KCl}$ solution filled straw, the RCRF coil and the normalized performance plots (yellow line on the image) using the reverse polarization method for transmit array system. a: The forward polarization mode of the image and the performance plot at the bottom, (b) the reverse polarization mode of the image and the performance plot at the bottom, and (c) the colorcoded image. Similar to the previous case shown in Fig. 5, the method successfully singles out the RCRF coil signal. Even though the disturber has higher signal intensity in the forward polarized mode, the reverse polarization method suppresses both the anatomy and the disturber significantly. [Color figure can be viewed in the online issue, which is available at wileyonlinelibrary.com.]

following imaging protocol: TR/TE: 40/3.4 ms, flip angle: $40^{\circ}$, slice thickness: $5 \mathrm{~mm}$. A Siemens $\mathrm{NaCl}$ solution phantom was used in phantom experiments.

The same ICRF coil and experiment setup were used in the volunteer experiments. The experiments conformed to the Guidelines for Experiments with Human Participants and were approved by the Bilkent University Ethics Committee, Ankara, Turkey.

\section{RESULTS}

\section{Catheter Tracking}

\section{Receive Array}

Figure 4 shows the oblique images of an oil phantom with two straws, one filled with $\mathrm{KCl}$ solution (left) and the other with the RCRF coil (right) inside. The results prove that the reverse polarization algorithm successfully suppresses both oil and $\mathrm{KCl}$ solution signals with receive (phased) array coils.

\section{Transmit Array}

Figure 5a shows a sagittal image of an oil phantom with two straws, one filled with $\mathrm{KCl}$ solution (left) and the other with the ICRF coil (right) inside. The results shows that the transmit array system enables the suppression of the phantom and $\mathrm{KCl}$ solution signals.

Next, the RCRF coil is used with the transmit array system. As shown in Fig. 6, the catheter still appears bright, suggesting flip angle amplification. This can be explained by the fact that the voltage across the diodes is the turn-on voltage and a small amount of current flows through the RCRF coil.

Figure 7 shows a transversal image of three straws, first filled with $\mathrm{KCl}$ solution (upper right straw), second

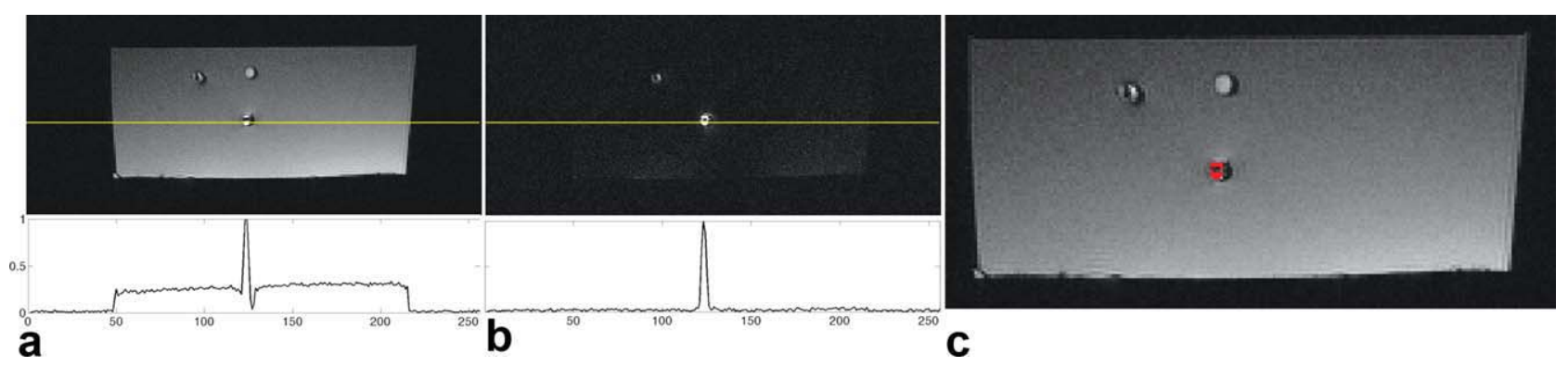

FIG. 7. Transversal images of the ICRF coil (on which the yellow line passes through), RCRF coil (the left dot above the yellow line), and KCI solution filled straw (top right dot above the yellow line) in the oil phantom with the normalized performance plots (yellow line on the image) using the reverse polarization method for transmit array system. a: The forward polarization mode of the image and the performance plot at the bottom, (b) the reverse polarization mode of the image and the performance plot at the bottom, and (c) the color-coded image. The reverse polarization method suppresses both the solution and the phantom. Both ICRF and RCRF coils are visible in the reverse polarization mode image. However, because of the transmission decoupling, the ICRF coil signal is stronger than the RCRF coil signal. [Color figure can be viewed in the online issue, which is available at wileyonlinelibrary.com.] 

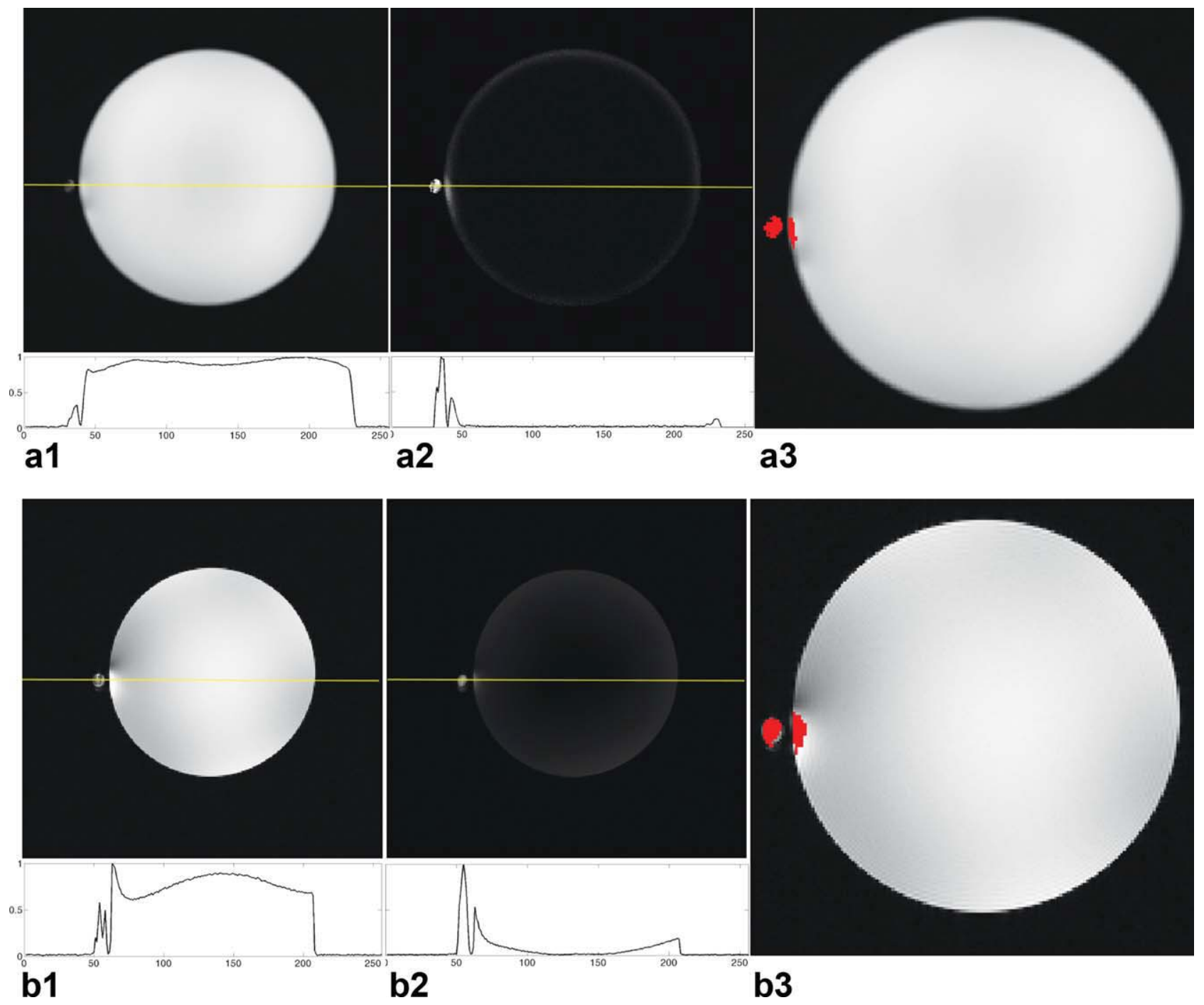

FIG. 8. a: Oblique image of the saline phantom and the normalized performance plots (yellow line on the image) using the reverse polarization method for phased array coil. (a1) The forward polarization mode of the image and the performance plot at the bottom, (a2) the reverse polarization mode of image and the performance plot at the bottom, and (a3) the color-coded image. b: Oblique image of the saline phantom with transmit array coil the normalized performance plots (yellow line on the image) using the reverse polarization method for transmit array system. (b1) The forward polarization mode of the image and the performance plot at the bottom, (b2) the reverse polarization mode of the image and the performance plot at the bottom, and (b3) the color-coded image. Although the phantom and contrast agent signals are stronger than the ICRF coil signal (left column) in forward polarized images for both receive and transmit array cases, the method suppresses all the signals other than the linearly polarized signal of the ICRF coil. [Color figure can be viewed in the online issue, which is available at wileyonlinelibrary.com.]

with the ICRF coil inside (lower straw), and third with the RCRF coil (upper left straw). Both the phantom and the KCl solutions were suppressed when the reverse polarization method was applied to the transmit array system while the ICRF and RCRF coil images were reconstructed. Note that, the ICRF coil signal is stronger due to transmit coupling.

\section{Fiducial Marker Visualization}

\section{Phantom Experiments}

Figure 8 shows sagittal images of a saline solution phantom with an ICRF coil attached to the phantom (Fig. 3). The reverse polarization algorithm successfully suppresses the phantom signal with phased array coil (Fig. 8a) and transmit array system (Fig. 8b). The color-coded images and the performance plots show that the algorithm works.

\section{Volunteer Experiments}

The transversal and sagittal images of receive and transmit array coils are shown in Figs. 9 and 10, respectively. Volunteer experiments are especially important to show the usefulness of the reverse polarization method for clinical applications. Furthermore, constructing the reverse polarized mode of a body birdcage coil is a challenge for $3 \mathrm{~T}$ transmit array systems. Demonstration of the efficiency is critical for higher fields in human studies (Figs. 9b and 10b) 

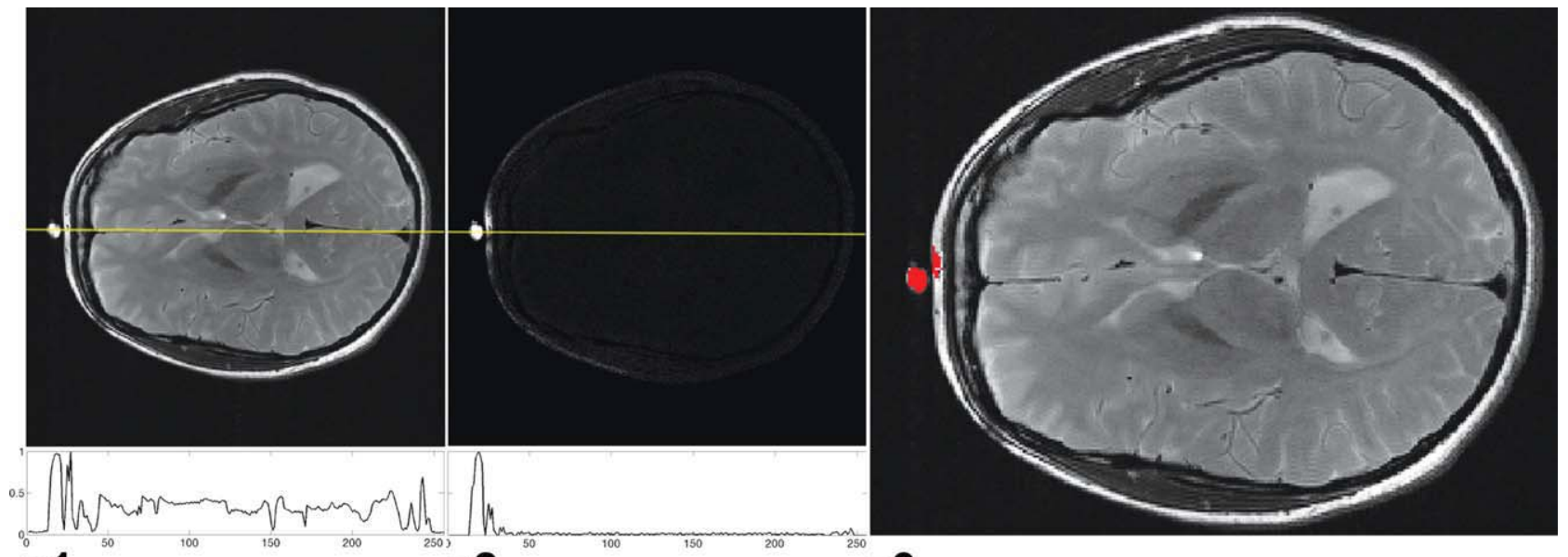

a1 a2 a3

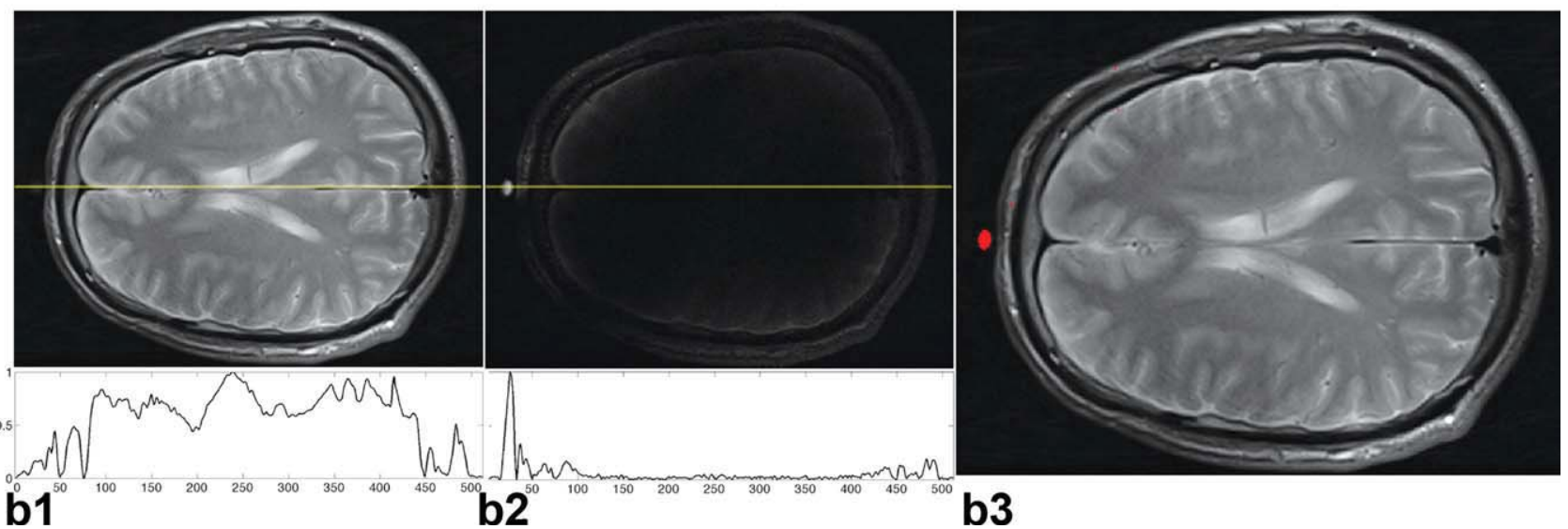

FIG. 9. a: Transversal image of the volunteer and the normalized performance plots (yellow line on the image) using the reverse polarization method for phased array coil. (a1) The forward polarization mode of the image and the performance plot at the bottom, (a2), the reverse polarization mode of the image and the performance plot at the bottom, and (a3) the color-coded image. b: Transversal image of the volunteer and the normalized performance plots (yellow line on the image) using the reverse polarization method for transmit array system. (b1) The forward polarization mode of the image and the performance plot at the bottom, (b2) the reverse polarization mode of the image and the performance plot at the bottom, and (b3) the color-coded image. [Color figure can be viewed in the online issue, which is available at wileyonlinelibrary.com.]

All of the figures presented in this section show that the reverse polarization method works successfully in both applications. The background signal is almost completely suppressed and the color-coded images enable visualization of the CRF coils.

\section{DISCUSSION}

Catheter tracking is necessary when conducting interventional procedures (22). Methods for tracking catheters can be divided into three main groups: passive, active, and hybrid. Paramagnetic materials (23-25), contrast agents (26-28), and other local signal manipulators $(29,30)$ are the main passive tracking tools. Passive tracking methods are simple to implement, but produce weak contrast. Active tracking methods solve the contrast issue (31-39) but cause safety and handling problems (22). Quick et al. (40) have introduced a hybrid technique using ICRF coils. The performance of the method depends on both the flip angle and the orientation of the ICRF coil. In this study, we have addressed this intrinsic problem of the coils.
An ICRF coil (41) is a simple wire loop resonated using a capacitor. These coils are used for small FOV imaging $(42,43)$ and catheter tracking $(1,40)$ to amplify transmit and receive signals in the vicinity. When a pair of back-to-back diodes is connected parallel to the resonating capacitor, transmission coupling is eliminated and only receptive coupling remains. Therefore, the RCRF coil is safer than the ICRF coil, because, negligible flip angle amplification occurs with the RCRF coil $(1,40)$. In this study, both ICRF and RCRF coils have been shown to be usable in catheter tracking and fiducial marker visualization applications.

As already known, a forward polarized transmission excites spins and coupled RF (CRF) coils, whereas, a reverse polarized transmission excites only CRF coils. With conventional imaging systems, only forward or reverse polarized transmission is possible. However, transmit array systems can switch between forward and reverse polarizations simultaneously. Transmit array studies are mostly concentrated on homogenizing higher frequency RF fields, but this causes another problem, namely excessive specific absorption rate. In this study, the Siemens' experimental eight- 


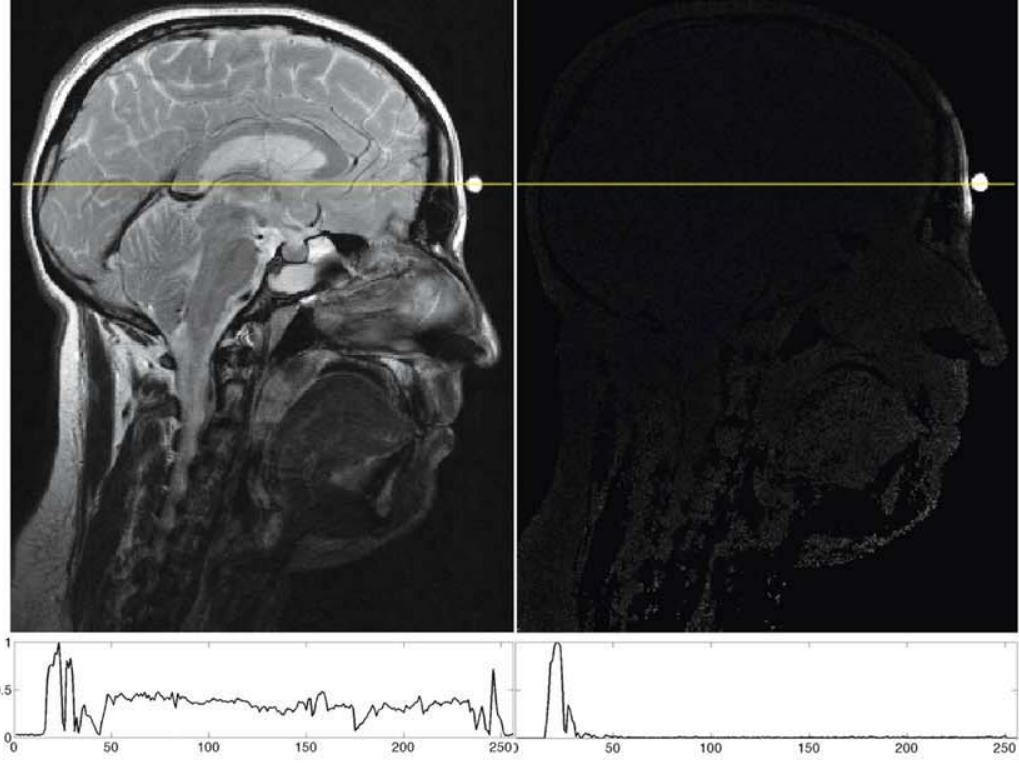

a1

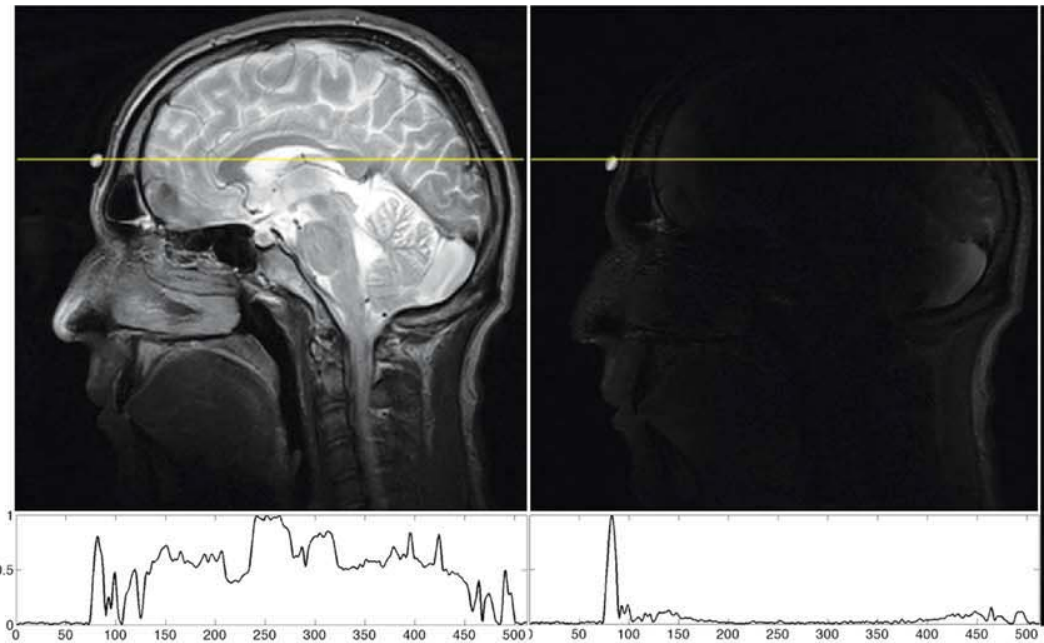

b1 b2

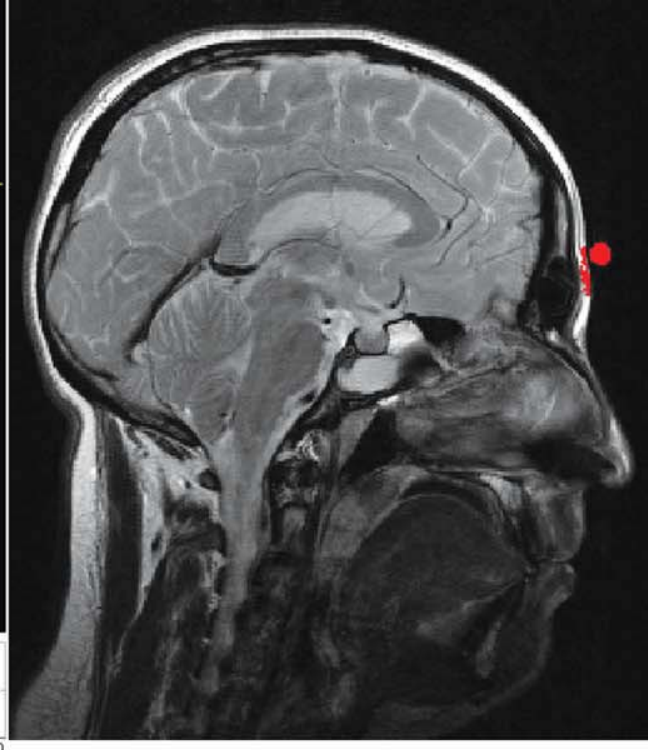

a3

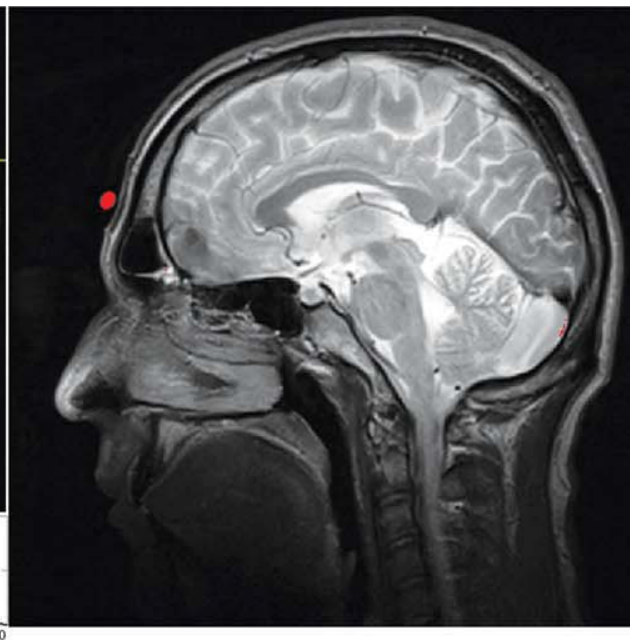

b3

FIG. 10. a: Sagittal image of the volunteer and the normalized performance plots (yellow line on the image) using the reverse polarization method for phased array coil. (a1) The forward polarization mode of the image and the performance plot at the bottom, (a2), the reverse polarization mode of the image and the performance plot at the bottom, and (a3) the color-coded image. b: Sagittal image of the volunteer and the normalized performance plots (yellow line on the image) using the reverse polarization method for transmit array system. (b1) The forward polarization mode of the image and the performance plot at the bottom, (b2) the reverse polarization mode of the image and the performance plot at the bottom, and (c3) the color-coded image. [Color figure can be viewed in the online issue, which is available at wileyonlinelibrary.com.]

channel transmit array system was used. The applications discussed in this article use only two channels and the phases and amplitudes of the RF waveforms are manipulated, but the shape of the waveforms were identical. Although the transmit array system's capabilities are not completely utilized, the body coil is much more accessible. It is already embedded in the imager and because it uses only two channels, it is simpler to work with.

Transmit and receive (phased) arrays offer separate excitation and reception profiles to improve the usability of the reverse polarization method. Here, the receive array coil data was processed to obtain a reverse polarization mode image, and the transmit array system was utilized to excite the CRF coil only, suppressing the anatomy signal.

In this study, the reverse polarization method was also implemented in fiducial marker visualization. During an invasive operation, a targeting procedure is vital. Fiducial markers are effectively used in different operations for registration purposes. However, in some sequences, these markers are not bright enough, so registering becomes more difficult. To improve the visibility of the markers, the reverse polarization method was implemented using CRF coils for image-guided surgery registration.

Performance of the reverse polarization method for phased array coils is directly related to the SNR of the 
coil. Therefore, the number of coil elements and their placement play an important role on the reverse polarization method performance similar to sensitivity based techniques. As the number of coils increases, reverse polarized region that can be obtained using this method is getting larger. For example, three-element phased array coil performs better than two-element coil when the elements have the same size.

Finally, the $x$-channel of the body coil was performing worse than the $y$-channel. To obtain the same signal strength from individual channels separately, the $y$-channel was multiplied by 0.67 , while the multiplier of the $x$-channel was kept at 1.00 . This problem was amplified by the dielectric effect (44). Therefore, oil was used in the catheter tracking phantom experiments, which helped to avoid dielectric effect-related inhomogeneities. However, carefully calibrating the channels resulted with proper reverse polarization mode. As a result, our initial tests on human volunteers produced enough homogeneity while using our $3 \mathrm{~T}$ scanner so that we were able to demonstrate the method in vivo.

\section{CONCLUSION}

In this study, the feasibility of the reverse polarization method was examined using transmit and receive array systems in two different applications of the method, i.e., catheter tracking and fiducial marker visualization. Using both transmit and receive RF coil arrays coupled to RCRF and ICRF coils, we showed that the method achieved successful background suppression in both phantom and volunteer studies.

\section{ACKNOWLEDGMENTS}

The authors are grateful to Volkan Acikel and Ozay Hirlakoglu for their supports during the experiments. They thank Prof. Ayhan Altintas and Emre Kopanoglu for their valuable discussions. They also thank Dr. Katja Dörschner, Dr. Aslihan Ors, and Gizem Kucukoglu for their help. Their sincere thanks are due to Dr. Arne Reykowski for his excellent advice on the mode matrix reconstruction and to Ms. Rana Nelson for her valuable editorial support.

\section{REFERENCES}

1. Celik H, Uluturk A, Tali T, Atalar E. A catheter tracking method using reverse polarization for MR-guided interventions. Magn Reson Med 2007;58:1224-1231.

2. Overall WR, Pauly JM, Stang PP, Scott GC. Ensuring safety of implanted devices under MRI using reversed RF polarization. Magn Reson Med 2010;64:823-833.

3. van den Brink JS, Rozijn TH, Smink J. Can reverse polarization be used to detect RF resonant structures at 3T? Stillwater, MN: ISMRM RF Safety Workshop.; 2010.

4. Quick H, Zenge M, Kuehl H, Kaiser G, Aker S, Eggebrecht H, Massing S, Ladd M. Wireless active catheter visualization: passive decoupling methods and their impact on catheter visibility. In: 13th Annual Meeting of ISMRM, Miami Beach, Florida, USA, 2005.

5. Celik H. Novel RF coil technologies for MRI. Ankara: Bilkent University; 2006. 97p.

6. Roemer PB, Edelstein WA, Hayes CE, Souza SP, Mueller OM. The NMR phased array. Magn Reson Med 1990;16:192-225.

7. Glover GH, Hayes CE, Pelc NJ, Edelstein WA, Mueller OM, Hart HR, Hardy CJ, O’Donnell M, Barber WD. Comparison of linear and circular polarization for magnetic resonance imaging. J Magn Reson 1985; 64:255-270.
8. Knufman NM, van den Elsen PA, Cillessen JP, van Isselt JW, Tulleken CA. Spatial integration of multimodal brain images in cerebral infarction. Brain Topogr 1992;5:165-169.

9. Maintz JA, Van den Elsen PA, Viergever MA. Registration of 3D medical images using simple morphological tools. Lecture Notes in Computer Science. Vol. 1230/1997: Heidelberg: Springer Berlin; 1997.

10. Maintz JB, Viergever MA. A survey of medical image registration. Med Image Anal 1998;2:1-36.

11. Van den Elsen PA, Maintz JA, Pol ED, Viergever MA. Automatic registration of CT and MR brain images using correlation of geometrical features. IEEE Trans Med Imaging 1995;14:384-396.

12. Viergever MA, Maintz JB, Niessen WJ, Noordmans HJ, Pluim JP, Stokking R, Vincken KL. Registration, segmentation, and visualization of multimodal brain images. Comput Med Imaging Graph 2001; 25:147-151.

13. Hayes CE, Edelstein WA, Schenck JF, Mueller OM, Eash M. An efficient, highly homogenous radiofrequency coil for whole-body NMR imaging at 1.5 T. J Magn Reson 1985;63:622-628.

14. Hoult DI. The principle of reciprocity in signal strength calculations - a mathematical guide. Concepts Magn Reson 2000;12:173-187.

15. Vesselle H, Collin RE. The signal-to-noise ratio of nuclear magnetic resonance surface coils and application to a lossy dielectric cylinder model. I. Theory. IEEE Trans Biomed Eng 1995;42:497-506.

16. Wright SM, Wald LL. Theory and application of array coils in MR spectroscopy. NMR Biomed 1997;10:394-410.

17. Reykowski A, Blasche M. Mode matrix-a generalized signal combiner for parallel imaging arrays. In: Proc. 12th Annual Meeting of the International Society for Magnetic Resonance in Medicine, Kyoto, 2004. p 1587.

18. Chen Z, Johnston LA, Kwon DH, Oh SH, Cho ZH, Egan GF. An optimised framework for reconstructing and processing MR phase images. Neuroimage 2010;49:1289-1300.

19. Cukur T, Lustig M, Nishimura DG. Multiple-profile homogeneous image combination: application to phase-cycled SSFP and multicoil imaging. Magn Reson Med 2008;60:732-738.

20. Pruessmann KP, Weiger M, Scheidegger MB, Boesiger P. SENSE: sensitivity encoding for fast MRI. Magn Reson Med 1999;42:952-962.

21. Cukur T, Santos JM, Pauly JM, Nishimura DG. Variable-density parallel imaging with partially localized coil sensitivities. IEEE Trans Med Imaging 2010;29:1173-1181.

22. Ratnayaka K, Faranesh AZ, Guttman MA, Kocaturk O, Saikus CE, Lederman RJ. Interventional cardiovascular magnetic resonance: still tantalizing. J Cardiovasc Magn Reson 2008;10:62.

23. Seppenwoolde JH, Viergever MA, Bakker CJ. Passive tracking exploiting local signal conservation: the white marker phenomenon. Magn Reson Med 2003;50:784-790.

24. Stuber M, Gilson WD, Schar M, Kedziorek DA, Hofmann LV, Shah S, Vonken EJ, Bulte JW, Kraitchman DL. Positive contrast visualization of iron oxide-labeled stem cells using inversion-recovery with ONresonant water suppression (IRON). Magn Reson Med 2007;58: 1072-1077.

25. Dharmakumar R, Koktzoglou I, Tang R, Harris KR, Beohar N, Li D. Off-resonance positive contrast imaging of a passive endomyocardial catheter in swine. Phys Med Biol 2008;53:N249-N257.

26. Edelman RR, Storey P, Dunkle E, Li W, Carrillo A, Vu A, Carroll TJ. Gadolinium-enhanced off-resonance contrast angiography. Magn Reson Med 2007;57:475-484.

27. Unal O, Li J, Cheng W, Yu H, Strother CM. MR-visible coatings for endovascular device visualization. J Magn Reson Imaging 2006;23: 763-769.

28. Krueger JJ, Ewert P, Yilmaz S, Gelernter D, Peters B, Pietzner K, Bornstedt A, Schnackenburg B, Abdul-Khaliq H, Fleck E, Nagel E, Berger F, Kuehne T. Magnetic resonance imaging-guided balloon angioplasty of coarctation of the aorta: a pilot study. Circulation 2006;113:1093-1100.

29. Miquel ME, Hegde S, Muthurangu V, Corcoran BJ, Keevil SF, Hill DL, Razavi RS. Visualization and tracking of an inflatable balloon catheter using SSFP in a flow phantom and in the heart and great vessels of patients. Magn Reson Med 2004;51:988-995.

30. Magnusson P, Johansson E, Mansson S, Petersson JS, Chai CM, Hansson G, Axelsson O, Golman K. Passive catheter tracking during interventional MRI using hyperpolarized 13C. Magn Reson Med 2007;57:1140-1147.

31. Atalar E, Bottomley PA, Ocali O, Correia LC, Kelemen MD, Lima JA, Zerhouni EA. High resolution intravascular MRI and MRS by using a catheter receiver coil. Magn Reson Med 1996;36:596-605. 
32. Bock M, Muller S, Zuehlsdorff S, Speier P, Fink C, Hallscheidt P, Umathum R, Semmler W. Active catheter tracking using parallel MRI and real-time image reconstruction. Magn Reson Med 2006;55: 1454-1459.

33. Buecker A, Adam GB, Neuerburg JM, Kinzel S, Glowinski A, Schaeffter T, Rasche V, van Vaals JJ, Guenther RW. Simultaneous real-time visualization of the catheter tip and vascular anatomy for MR-guided PTA of iliac arteries in an animal model. J Magn Reson Imaging 2002;16:201-208.

34. Dumoulin CL, Souza SP, Darrow RD. Real-time position monitoring of invasive devices using magnetic resonance. Magn Reson Med 1993;29:411-415.

35. Feng L, Dumoulin CL, Dashnaw S, Darrow RD, Guhde R, Delapaz RL, Bishop PL, Pile-Spellman J. Transfemoral catheterization of carotid arteries with real-time MR imaging guidance in pigs. Radiology 2005;234:551-557.

36. Hillenbrand CM, Elgort DR, Wong EY, Reykowski A, Wacker FK, Lewin JS, Duerk JL. Active device tracking and high-resolution intravascular MRI using a novel catheter-based, opposed-solenoid phased array coil. Magn Reson Med 2004;51:668-675.

37. Ocali O, Atalar E. Intravascular magnetic resonance imaging using a loopless catheter antenna. Magn Reson Med 1997;37:112-118.
38. Schirra CO, Weiss S, Krueger S, Pedersen SF, Razavi R, Schaeffter T, Kozerke S. Toward true $3 \mathrm{D}$ visualization of active catheters using compressed sensing. Magn Reson Med 2009;62:341-347.

39. Zuehlsdorff S, Umathum R, Volz S, Hallscheidt P, Fink C, Semmler W, Bock M. MR coil design for simultaneous tip tracking and curvature delineation of a catheter. Magn Reson Med 2004;52:214-218.

40. Quick HH, Zenge MO, Kuehl H, Kaiser G, Aker S, Massing S, Bosk $\mathrm{S}$, Ladd ME. Interventional magnetic resonance angiography with no strings attached: wireless active catheter visualization. Magn Reson Med 2005;53:446-455.

41. Froncisz W, Jesmanowicz A, Hyde JS. Inductive (Flux linkage) coupling to local coils in magnetic-resonance-imaging and spectroscopy. J Magn Reson 1986;66:135-143.

42. Bilgen M, Al-Hafez B, He YY, Brooks WM. Magnetic resonance angiography of rat spinal cord at 9.4 T: a feasibility study. Magn Reson Med 2005;53:1459-1461.

43. Quick HH, Kuehl H, Kaiser G, Bosk S, Debatin JF, Ladd ME. Inductively coupled stent antennas in MRI. Magn Reson Med 2002;48:781-790.

44. Ibrahim TS, Lee R, Abduljalil AM, Baertlein BA, Robitaille P-ML. Dielectric resonances and B1 field inhomogeneity in UHFMRI: computational analysis and experimental findings. Magn Reson Imaging 2001;19:219-226 